PROCEEDINGS OF THE

AMERICAN MATHEMATICAL SOCIETY

Volume 136, Number 10, October 2008, Pages 3461-3466

S 0002-9939(08)09420-3

Article electronically published on May 19, 2008

\title{
NEW CHARACTERIZATION OF $\Sigma$-INJECTIVE MODULES
}

\author{
K. I. BEIDAR, S. K. JAIN, AND ASHISH K. SRIVASTAVA \\ (Communicated by Birge Huisgen-Zimmermann)
}

\begin{abstract}
We provide a new characterization for an injective module to be $\Sigma$-injective.
\end{abstract}

\section{INTRODUCTION}

In his paper 4, Carl Faith introduced the concept of $\Sigma$-injectivity and defined an injective module $M$ to be $\Sigma$-injective if every direct sum of copies of $M$ is injective. It turns out that such an $R$-module $M$ provides a good deal of information about the structure of a ring $R$. For example, $R$ is right noetherian if and only if every injective right $R$-module is $\Sigma$-injective [5]. If $R$ is an integral domain, then the injective hull $E\left(R_{R}\right)$ of $R$ is $\Sigma$-injective if and only if $R$ is a right Ore domain [4]. Goursaud-Valette showed that if a ring $R$ admits a faithful $\Sigma$-injective module, then $R$ is a right Goldie ring [6].

The following characterizations are well-known for an injective module to be $\Sigma$-injective.

Theorem 1 (Cailleau [3], Faith [4). For an injective module $M_{R}$, the following are equivalent:

(1) $M$ is $\Sigma$-injective.

(2) $M$ is countably $\Sigma$-injective.

(3) $R$ satisfies ACC on the set of right ideals $I$ of $R$ that are annihilators of subsets of $M$.

(4) $M$ is a direct sum of indecomposable $\Sigma$-injective modules.

The purpose of this paper is to provide the following new characterization for an injective module to be $\Sigma$-injective.

Theorem 2. Let $M_{R}$ be an injective module. Then the following statements are equivalent:

(a) $M$ is $\Sigma$-injective.

(b) Every essential extension of $M^{\left(\aleph_{0}\right)}$ is a direct sum of injective modules.

Received by the editors July 16, 2006, and, in revised form, February 12, 2007, May 24, 2007, July 27, 2007, and September 15, 2007.

2000 Mathematics Subject Classification. Primary 16D50, 16P40.

Key words and phrases. Injective modules, $\Sigma$-injective modules, essential extensions, right noetherian rings.

The first author is deceased.

(C)2008 American Mathematical Society Reverts to public domain 28 years from publication 


\section{Preliminaries}

All rings considered in this paper have unity, and all modules are right unital. We denote by $E(M)$ the injective hull of $M$. We shall write $N \subseteq_{e} M$ whenever $N$ is an essential submodule of $M$. A submodule $L$ of $M$ is called an essential closure of a submodule $N$ of $M$ if it is a maximal essential extension of $N$ in $M$. A submodule $K$ of $M$ is called a complement if there exists a submodule $U$ of $M$ such that $K$ is maximal with respect to the property that $K \cap U=0$. Given a cardinal $\alpha$ and a module $N$, we denote by $N^{(\alpha)}$ the direct sum of $\alpha$ copies of the module $N$. A module $N$ is said to be $\Sigma$-injective provided that $N^{(\alpha)}$ is injective for any cardinal $\alpha$. We say that the Goldie dimension $G \operatorname{dim}_{U}(N)$ of $N$ with respect to $U$ is finite, written as $G \operatorname{dim}_{U}(N)<\infty$, if $N$ does not contain an infinite independent family of nonzero submodules which are isomorphic to submodules of $U$. A module $N$ is said to be q.f.d. relative to $U$ if for any factor module $\bar{N}$ of $N, G \operatorname{dim}_{U}(\bar{N})<\infty$. We say $R$ is right q.f.d. relative to $U$ if $R_{R}$ is q.f.d. relative to $U$.

We first start with a key lemma.

Lemma 3. Let $M$ be an injective module and suppose that every essential extension of $M^{\left(\aleph_{0}\right)}$ is a direct sum of injective modules. Then

(a) Given a direct sum $G=\bigoplus_{i \in \mathbb{N}} M_{i}, M_{i} \cong M$, and nonzero injective submodules $V_{i}$ of $M_{i}$, there exists an infinite subset $\mathcal{J} \subseteq \mathbb{N}$ and nonzero injective submodules $V_{j}^{\prime} \subseteq V_{j}, j \in \mathcal{J}$, such that $\bigoplus_{j \in \mathcal{J}} V_{j}^{\prime}$ is injective.

In particular, if $\left\{V_{i}: i \in \mathbb{N}\right\}$ is an independent family of uniform injective submodules of $M$, then $\bigoplus_{j \in \mathcal{J}} V_{j}$ is injective for some infinite subset $\mathcal{J} \subseteq \mathbb{N}$.

(b) $R$ is right q.f.d. relative to $M$.

Proof. (a) Set $E=E(G)$. Since $V_{i}$ is an injective submodule of $M_{i}, M_{i}=V_{i} \oplus M_{i}^{\prime}$ for some submodule $M_{i}^{\prime} \subseteq M_{i}$. Therefore, $G=\left(\bigoplus_{i \in \mathbb{N}} V_{i}\right) \oplus\left(\bigoplus_{i \in \mathbb{N}} M_{i}^{\prime}\right)$. Let $H$ and $H^{\prime}$ be essential closures of $\bigoplus_{i \in \mathbb{N}} V_{i}$ and $\bigoplus_{i \in \mathbb{N}} M_{i}^{\prime}$ in $E$, respectively. Clearly, $E=H \oplus H^{\prime}$. If $\bigoplus_{i \in \mathbb{N}} V_{i}=H$, then there is nothing to prove.

Now consider the case when $\bigoplus_{i \in \mathbb{N}} V_{i} \neq H$. Pick $x \in H \backslash \bigoplus_{i \in \mathbb{N}} V_{i}$. Let $Q$ be a submodule of $H$ maximal with respect to the properties that $\bigoplus_{i \in \mathbb{N}} V_{i} \subseteq Q$ and $x \notin Q$. Set $P=Q \oplus H^{\prime}$ and note that $E / P=\left(H \oplus H^{\prime}\right) /\left(Q \oplus H^{\prime}\right) \cong H / Q$ is a subdirectly irreducible module.

Now, as $G \subseteq_{e} E$ and $G \subseteq P \subset E$, we have $G \subseteq_{e} P$. Hence, by our assumption, $P=\bigoplus_{k \in \mathcal{K}} W_{k}$, where each $W_{k}$ is a nonzero injective module. Since $P \subset_{e} E$ and $P \neq E, P$ is not injective, and so $|\mathcal{K}|=\infty$.

We claim that for any finite subset $\mathcal{L}$ of $\mathcal{K}$ and for any positive integer $n$ there exists $i>n$ such that $V_{i} \cap\left(\bigoplus_{k \in \mathcal{L}} W_{k}\right)$ is not essential in $V_{i}$.

Suppose the above claim is not true. Then there exists a finite subset $\mathcal{L} \subseteq \mathcal{K}$ and an integer $n \geq 1$ such that $V_{i} \cap\left(\bigoplus_{k \in \mathcal{L}} W_{k}\right) \subset_{e} V_{i}$ for all $i>n$. Let $A$ be an essential closure of $\bigoplus_{i>n}\left(V_{i} \cap\left(\bigoplus_{k \in \mathcal{L}} W_{k}\right)\right)$ in $\bigoplus_{k \in \mathcal{L}} W_{k}$ which is injective, and so $A$ is also injective.

We have $\bigoplus_{i>n}\left(V_{i} \cap \bigoplus_{k \in \mathcal{L}} W_{k}\right) \subset_{e} A \subset \bigoplus_{k \in \mathcal{L}} W_{k}$. Setting $B=V_{1} \oplus V_{2} \oplus \ldots \oplus$ $V_{n} \oplus A$, we have $V_{1} \oplus V_{2} \oplus \ldots \oplus V_{n} \oplus_{i>n}\left(V_{i} \cap \bigoplus_{k \in \mathcal{L}} W_{k}\right) \subset_{e} B \subset E=H \oplus H^{\prime}$. Now, $\left(\left(\bigoplus_{i \leq n} V_{i}\right) \oplus_{i>n}\left(V_{i} \cap\left(\bigoplus_{k \in \mathcal{L}} W_{k}\right)\right)\right) \cap H \subset_{e} B \cap H \subset H$, which gives $\left(\bigoplus_{i \leq n} V_{i}\right) \oplus_{i>n}$ $\left(V_{i} \cap\left(\bigoplus_{k \in \mathcal{L}} W_{k}\right)\right) \subset_{e} B \cap H \subset H$. Since $V_{i} \cap\left(\bigoplus_{k \in \mathcal{L}} W_{k}\right) \subset_{e} V_{i}$ for all $i>n$, we have $\left(\bigoplus_{i \leq n} V_{i}\right) \oplus_{i>n}\left(V_{i} \cap\left(\bigoplus_{k \in \mathcal{L}} W_{k}\right)\right) \subset_{e} \bigoplus_{i \in \mathbb{N}} V_{i} \subset_{e} H$. Thus $B \cap H$ is an essential 
submodule of $H$. Furthermore, as $\left(\bigoplus_{i \leq n} V_{i}\right) \oplus_{i>n}\left(V_{i} \cap\left(\bigoplus_{k \in \mathcal{L}} W_{k}\right)\right) \subset_{e} B$, we have $B \cap H \subset_{e} B$.

Since $B \cap H \subset_{e} B$, we have $B \cap H^{\prime}=0$. As $B \cap H \subset_{e} H$, we have $(B \cap H) \oplus H^{\prime} \subset_{e}$ $H \oplus H^{\prime}=E$. Therefore, $B \oplus H^{\prime} \subset_{e} E$. But since both $B$ and $H^{\prime}$ are injective, $B \oplus H^{\prime}$ is injective. Thus $E=B \oplus H^{\prime}=\left(V_{1} \oplus V_{2} \oplus \ldots \oplus V_{n} \oplus A\right) \oplus H^{\prime} \subseteq Q+P+H^{\prime}=P$, a contradiction because $P \subset E$ and $P \neq E$.

This proves that for any finite subset $\mathcal{L}$ of $\mathcal{K}$ and for any positive integer $n$ there exists $i>n$ such that $V_{i} \cap\left(\bigoplus_{k \in \mathcal{L}} W_{k}\right)$ is not essential in $V_{i}$.

We now proceed by induction to construct a sequence of submodules $\left\{W_{k_{j}}^{\prime}\right.$ : $j=1,2, \ldots, n, \ldots\}$ such that each $W_{k_{j}}^{\prime}$ is a nonzero injective submodule of $W_{k_{j}}$ isomorphic to a submodule $V_{i_{j}}^{\prime}$ of $V_{i_{j}}$, where $k_{1}, k_{2}, \ldots, k_{n}, \ldots$ are distinct elements of $\mathcal{K}$ and $1 \leq i_{1}<i_{2}<\ldots<i_{n}<\ldots$

Let $i_{1} \geq 1$ be arbitrary. Now $V_{i_{1}} \subset \bigoplus_{k \in \mathcal{K}} W_{k}$ implies that there exists a nonzero submodule $V_{i_{1}}^{\prime}$ of $V_{i_{1}}$ such that $V_{i_{1}}^{\prime}$ is isomorphic to a submodule $W_{k_{1}}^{\prime}$ of $W_{k_{1}}$ for some $k_{1} \in \mathcal{K}$. Clearly, we may choose $V_{i_{1}}^{\prime}$ to be an injective submodule of $V_{i_{1}}$.

For $n \geq 1$, assume that we have a sequence $\left\{W_{k_{j}}^{\prime}: j=1,2, \ldots, n\right\}$ with the above stated property. By the fact proved above, there exists $i_{n+1}>i_{n}$ such that $X=V_{i_{n+1}} \cap\left(\bigoplus_{k \in \mathcal{K}_{1}} W_{k}\right)$ is not essential in $V_{i_{n+1}}$, where $\mathcal{K}_{1}=\left\{k_{1}, k_{2}, \ldots, k_{n}\right\}$. Let $X^{\prime}$ be a complement of $X$ in $V_{i_{n+1}}$. Then $X^{\prime} \neq 0$ and $X^{\prime} \cap\left(\bigoplus_{k \in \mathcal{K}_{1}} W_{k}\right)=$ $X^{\prime} \cap X=0$. We have $X^{\prime} \subset V_{i_{n+1}} \subset\left(\bigoplus_{k \in \mathcal{K}_{1}} W_{k}\right) \oplus\left(\bigoplus_{k \in \mathcal{K}_{2}} W_{k}\right)$, where $\mathcal{K}_{2}=$ $\mathcal{K} \backslash \mathcal{K}_{1}$. Let $\pi:\left(\bigoplus_{k \in \mathcal{K}_{1}} W_{k}\right) \oplus\left(\bigoplus_{k \in \mathcal{K}_{2}} W_{k}\right) \longrightarrow \bigoplus_{k \in \mathcal{K}_{2}} W_{k}$ be the projection. Then $\operatorname{ker}\left(\left.\pi\right|_{X^{\prime}}\right)=X^{\prime} \cap\left(\bigoplus_{k \in \mathcal{K}_{1}} W_{k}\right)=0$. Therefore, $X^{\prime}$ is isomorphic to some submodule of $\bigoplus_{k \in \mathcal{K}_{2}} W_{k}$. So, $X^{\prime}$ contains a nonzero submodule which is isomorphic to a submodule $F$ of $W_{k_{n+1}}$ for some $k_{n+1} \in \mathcal{K}_{2}$. Denote by $W_{k_{n+1}}^{\prime}$ an essential closure of $F$ in $W_{k_{n+1}}$. Since $F$ is isomorphic to a submodule of the injective module $V_{i_{n+1}}$, we conclude that $W_{k_{n+1}}^{\prime}$ is isomorphic to a submodule of $V_{i_{n+1}}$ as well. Obviously the family $\left\{W_{k_{j}}^{\prime}: j=1,2, \ldots, n+1\right\}$ satisfies the required property. This completes the induction argument.

Now set $\mathcal{K}^{\prime}=\left\{k_{1}, k_{2}, \ldots, k_{n}, \ldots\right\}$. Choose disjoint subsets $\mathcal{K}_{1}^{\prime}$ and $\mathcal{K}_{2}^{\prime}$ of $\mathcal{K}$ such that $\mathcal{K}=\mathcal{K}_{1}^{\prime} \cup \mathcal{K}_{2}^{\prime}$ and $\mathcal{K}^{\prime} \cap \mathcal{K}_{1}^{\prime}=\left\{k_{1}, k_{3}, \ldots, k_{2 n+1}, \ldots\right\}$. Clearly, $\mathcal{K}^{\prime} \cap \mathcal{K}_{2}^{\prime}=$ $\left\{k_{2}, k_{4}, \ldots, k_{2 n}, \ldots\right\}$.

Now we claim that either $\bigoplus_{k \in \mathcal{K}_{1}^{\prime}} W_{k}$ is injective or $\bigoplus_{k \in \mathcal{K}_{2}^{\prime}} W_{k}$ is injective.

Set $V=\bigoplus_{k \in \mathcal{K}_{1}^{\prime}} W_{k}$ and $W=\bigoplus_{k \in \mathcal{K}_{2}^{\prime}} W_{k}$. We have $P=V \oplus W$. Let $\widehat{V}$ and $\widehat{W}$ be essential closures of $V$ and $W$, respectively, in $E$. Clearly, $E=\widehat{V} \oplus \widehat{W}$. Therefore, $E / P=(\widehat{V} \oplus \widehat{W}) /(V \oplus W) \cong(\widehat{V} / V) \times(\widehat{W} / W)$. Since $E / P$ is shown to be subdirectly irreducible in the beginning of the proof, we have either $V=\widehat{V}$ or $W=\widehat{W}$. This proves our claim.

Thus, we may assume, without loss of generality, that the module $\bigoplus_{k \in \mathcal{K}_{1}^{\prime}} W_{k}$ is injective. Since $\bigoplus_{n=0}^{\infty} W_{k_{2 n+1}}^{\prime}$ is a direct summand of $\bigoplus_{k \in \mathcal{K}_{1}^{\prime}} W_{k}$, we get that $\bigoplus_{n=0}^{\infty} W_{k_{2 n+1}}^{\prime}$ is injective. Recalling that $\bigoplus_{n=0}^{\infty} V_{i_{2 n+1}}^{\prime} \cong \bigoplus_{n=0}^{\infty} W_{k_{2 n+1}}^{\prime}$, we conclude that $\bigoplus_{n=0}^{\infty} V_{i_{2 n+1}}^{\prime}$ is an injective module. This completes the proof.

(b) Assume to the contrary that $R$ is not right q.f.d. relative to $M$. Then there exists a cyclic right $R$-module $C$ with an infinite independent family $\left\{C_{i}: i \in \mathbb{N}\right\}$ of 
nonzero submodules of $C$ such that each $C_{i}$ is isomorphic to a submodule $B_{i}$ of $M$. Set $D_{i}$ equal to an essential closure of $B_{i}$ in $M$. Then $\left\{D_{i}: i \in \mathbb{N}\right\}$ is a family of injective submodules of $M$. Therefore by (a), there exists an infinite subset $\mathcal{J} \subseteq \mathbb{N}$ and nonzero injective submodules $D_{j}^{\prime} \subseteq D_{j}, j \in \mathcal{J}$, such that $\bigoplus_{j \in \mathcal{J}} D_{j}^{\prime}$ is injective. Set $B_{j}^{\prime}=B_{j} \cap D_{j}^{\prime}, j \in \mathcal{J}$ and note that $B_{j}^{\prime} \neq 0$. Let $C_{j}^{\prime}$ be the inverse image of $B_{j}^{\prime}$ under the isomorphism $C_{j} \longrightarrow B_{j}$ stated above. This induces a canonical isomorphism between $\bigoplus_{j \in \mathcal{J}} C_{j}^{\prime}$ and $\bigoplus_{j \in \mathcal{J}} B_{j}^{\prime}$, say $\theta$. Let $\sigma$ be the inclusion map $\bigoplus_{j \in \mathcal{J}} B_{j}^{\prime} \longrightarrow \bigoplus_{j \in \mathcal{J}} D_{j}^{\prime}$. Then, since $\bigoplus_{j \in \mathcal{J}} D_{j}^{\prime}$ is injective, the map $f=\sigma \theta$ : $\bigoplus_{j \in \mathcal{J}} C_{j}^{\prime} \longrightarrow \bigoplus_{j \in \mathcal{J}} D_{j}^{\prime}$ can be extended to a homomorphism $\widehat{f}: C \longrightarrow \bigoplus_{j \in \mathcal{J}} D_{j}^{\prime}$. Because $C$ is cyclic, there exists a finite subset $\mathcal{K} \subseteq \mathcal{J}$ such that $\widehat{f}(C) \subseteq \bigoplus_{k \in \mathcal{K}} D_{k}^{\prime}$. Now, $\widehat{f}\left(C_{j}^{\prime}\right)=f\left(C_{j}^{\prime}\right)=\sigma \theta\left(C_{j}^{\prime}\right)=\sigma\left(B_{j}^{\prime}\right)=B_{j}^{\prime}$. But $\widehat{f}\left(C_{j}^{\prime}\right) \subseteq \widehat{f}(C) \cap D_{j}^{\prime}=0$ for all $j \notin \mathcal{K}$, a contradiction.

Therefore, $R$ is right q.f.d. relative to $M$.

\section{Proof of Theorem 2}

Proof. (b) $\Longrightarrow$ (a). Suppose that $M^{(\lambda)}$ is not injective for some infinite cardinal $\lambda$. Set $E=E\left(M^{(\lambda)}\right)$, pick $x \in E \backslash M^{(\lambda)}$ and let $L=x R$. By Lemma 3 (b), $R$ is right q.f.d. relative to $M$. From this it follows that every nonzero cyclic and hence every nonzero submodule of $M$ contains a uniform submodule. Now, consider the set $\mathcal{S}$ of independent families $\left(M_{k}\right)_{k \in \mathcal{K}}$ of uniform injective modules $0 \neq M_{k} \subseteq M$. Suppose $\mathcal{S}$ is partially ordered by $\left(M_{k}\right)_{k \in \mathcal{K}} \leq\left(N_{l}\right)_{l \in \mathcal{L}}$ if and only if $\mathcal{K} \subseteq \mathcal{L}$ and $M_{k}=N_{k}$ for $k \in \mathcal{K}$. By Zorn's lemma we get a maximal independent family $\left(M_{i}\right)_{i \in \mathcal{I}}$ of uniform injective submodules. Clearly $\bigoplus_{i \in \mathcal{I}} M_{i} \subseteq_{e} M$, because otherwise we will get a contradiction to the maximality of this independent family of submodules. This yields that we have an independent family $\left\{W_{i}: i \in \mathcal{I}\right\}$ of uniform injective submodules of $M^{(\lambda)}$ such that each $W_{i}$ is isomorphic to a submodule of $M$ and $\bigoplus_{i \in \mathcal{I}} W_{i} \subseteq_{e} M^{(\lambda)}$.

Now we proceed to show that there is a sequence of pairwise distinct elements $i_{1}, i_{2}, \ldots$ in $\mathcal{I}$ and an independent family of direct summands $V_{1}, V_{2}, \ldots$ of $E$ such that $V_{j} \cong W_{i_{j}}$ with $V_{j} \oplus\left(\bigoplus_{i \in \mathcal{I}_{j}} W_{i}\right)=\bigoplus_{i \in \mathcal{I}_{j-1}} W_{i}, E=E_{j} \oplus\left(\bigoplus_{k=1}^{j} V_{k}\right)$ and $\pi_{j-1}(L) \cap V_{j} \neq 0$ for all $j \in \mathbb{N}$, where $\mathcal{I}_{0}=\mathcal{I}, \mathcal{I}_{j}=\mathcal{I}_{j-1} \backslash\left\{i_{j}\right\}$ for $i_{j} \in \mathcal{I}, E_{0}=E$, $E_{j}$ is an essential closure of $\bigoplus_{i \in \mathcal{I}_{j}} W_{i}$ in $E_{j-1}, \pi_{0}=i d_{E}$, and $\pi_{j}$ is the projection of $E$ onto $E_{j}$ along $V_{1} \oplus \ldots \oplus V_{j}$.

Since $\bigoplus_{i \in \mathcal{I}} W_{i} \subseteq_{e} M^{(\lambda)} \subset_{e} E$ and $L$ is a nonzero submodule of $E$, we have $L \cap\left(\bigoplus_{i \in \mathcal{I}} W_{i}\right) \neq 0$. So $L \cap\left(\bigoplus_{i \in \mathcal{I}} W_{i}\right)$ contains a nonzero cyclic uniform submodule, say, $C_{1}$. This implies that there exists a finite subset $\mathcal{K}_{1} \subset \mathcal{I}$ such that $C_{1} \subseteq$ $\bigoplus_{i \in \mathcal{K}_{1}} W_{i}$. Let $V_{1}$ be an essential closure of $C_{1}$ in $\bigoplus_{i \in \mathcal{K}_{1}} W_{i}$. Since $\bigoplus_{i \in \mathcal{K}_{1}} W_{i}$ is injective, $V_{1}$ is injective. So, $\bigoplus_{i \in \mathcal{K}_{1}} W_{i}=V_{1} \oplus D_{1}$ for some submodule $D_{1}$ of $\bigoplus_{i \in \mathcal{K}_{1}} W_{i}$. Since $V_{1}$ is injective, it has the exchange property. Therefore, $\bigoplus_{i \in \mathcal{K}_{1}} W_{i}=V_{1} \oplus\left(\bigoplus_{i \in \mathcal{K}_{1}} W_{i}^{\prime}\right)$ for some submodules $W_{i}^{\prime}$ of $W_{i}$. Since $W_{i}^{\prime}$ are injective and each $W_{i}$ is indecomposable, either $W_{i}^{\prime}=0$ or $W_{i}^{\prime}=W_{i}$. We recall that $V_{1}$ is uniform because it is the closure of the uniform module $C_{1}$. Comparing the Goldie dimension on each side of $\bigoplus_{i \in \mathcal{K}_{1}} W_{i}=V_{1} \oplus\left(\bigoplus_{i \in \mathcal{K}_{1}} W_{i}^{\prime}\right)$, we get that there exists exactly one index, say $i_{1} \in \mathcal{K}_{1}$, such that $W_{i_{1}}^{\prime}=0$, and for all $i\left(\neq i_{1}\right) \in \mathcal{K}_{1}, W_{i}^{\prime}=W_{i}$. So, $\bigoplus_{i \in \mathcal{K}_{1}} W_{i}=V_{1} \oplus\left(\bigoplus_{i \in \mathcal{K}_{1} \backslash\left\{i_{1}\right\}} W_{i}\right)$. This yields 
$V_{1} \cong\left(\bigoplus_{i \in \mathcal{K}_{1}} W_{i}\right) /\left(\bigoplus_{i \in \mathcal{K}_{1} \backslash\left\{i_{1}\right\}} W_{i}\right) \cong W_{i_{1}}$. Also, we have $V_{1} \oplus\left(\bigoplus_{i \in \mathcal{K}_{1} \backslash\left\{i_{1}\right\}} W_{i}\right) \oplus$ $\left(\bigoplus_{i \in \mathcal{I} \backslash \mathcal{K}_{1}} W_{i}\right)=\left(\bigoplus_{i \in \mathcal{K}_{1}} W_{i}\right) \oplus\left(\bigoplus_{i \in \mathcal{I} \backslash \mathcal{K}_{1}} W_{i}\right)$. This yields $V_{1} \oplus\left(\bigoplus_{i \in \mathcal{I}_{1}} W_{i}\right)=$ $\bigoplus_{i \in \mathcal{I}} W_{i}$. Taking injective hulls of both sides, we get $E_{1} \oplus V_{1}=E$. Clearly, $L \cap V_{1} \neq 0$ as it contains $C_{1}$.

For $n \geq 1$, assume that we have a sequence $\left\{V_{j}\right\}, 1 \leq j \leq n$, of submodules of $E$ with the above stated properties. Since $x \notin M^{(\lambda)}, L=x R \nsubseteq \bigoplus_{i=1}^{n} V_{i}=\operatorname{ker}\left(\pi_{n}\right)$, if $x \in \bigoplus_{i=1}^{n} V_{i}$, then $V_{1} \oplus \ldots \oplus V_{n} \oplus\left(\bigoplus_{i \in \mathcal{I}_{n}} W_{i}\right)=\bigoplus_{i \in \mathcal{I}_{0}} W_{i}$ implies that $x$ belongs to $\bigoplus_{i \in \mathcal{I}_{0}} W_{i}$ and hence to $M^{(\lambda)}$, a contradiction. So $\pi_{n}(L) \neq 0$. Now $\bigoplus_{i \in \mathcal{I}_{n}} W_{i} \subset_{e} E_{n}$ and because $\pi_{n}: E \longrightarrow E_{n}$, we have $\pi_{n}(L) \cap\left(\bigoplus_{i \in \mathcal{I}_{n}} W_{i}\right) \neq 0$. So $\pi_{n}(L) \cap\left(\bigoplus_{i \in \mathcal{I}_{n}} W_{i}\right)$ contains a nonzero cyclic uniform submodule, say, $C_{n+1}$. This implies, there exists a finite subset $\mathcal{K}_{n+1} \subseteq \mathcal{I}_{n}$ such that $C_{n+1} \subseteq \bigoplus_{i \in \mathcal{K}_{n+1}} W_{i}$. Let $V_{n+1}$ be an essential closure of $C_{n+1}$ in $\bigoplus_{i \in \mathcal{K}_{n+1}} W_{i}$. Since $\bigoplus_{i \in \mathcal{K}_{n+1}} W_{i}$ is injective, $V_{n+1}$ is injective. So, $\bigoplus_{i \in \mathcal{K}_{n+1}} W_{i}=V_{n+1} \oplus D_{n+1}$ for some submodule $D_{n+1}$ of $\bigoplus_{i \in \mathcal{K}_{n+1}} W_{i}$. Since $V_{n+1}$ is injective, it has the exchange property. Therefore, $\bigoplus_{i \in \mathcal{K}_{n+1}} W_{i}=V_{n+1} \oplus\left(\bigoplus_{i \in \mathcal{K}_{n+1}} W_{i}^{\prime}\right)$ for some submodules $W_{i}^{\prime}$ of $W_{i}$. Since $W_{i}^{\prime}$ are injective and each $W_{i}$ is indecomposable, either $W_{i}^{\prime}=0$ or $W_{i}^{\prime}=W_{i}$. Again note that $V_{n+1}$ is uniform because it is the closure of the uniform module $C_{n+1}$. Comparing the Goldie dimension on each side of $\bigoplus_{i \in \mathcal{K}_{n+1}} W_{i}=V_{n+1} \oplus$ $\left(\bigoplus_{i \in \mathcal{K}_{n+1}} W_{i}^{\prime}\right)$, we get that there exists exactly one index, say $i_{n+1} \in \mathcal{K}_{n+1}$, such that $W_{i_{n+1}}^{\prime}=0$, and for all $i\left(\neq i_{n+1}\right) \in \mathcal{K}_{n+1}, W_{i}^{\prime}=W_{i}$. So, $\bigoplus_{i \in \mathcal{K}_{n+1}} W_{i}=V_{n+1} \oplus$ $\left(\bigoplus_{i \in \mathcal{K}_{n+1} \backslash\left\{i_{n+1}\right\}} W_{i}\right)$. This yields $V_{n+1} \cong\left(\bigoplus_{i \in \mathcal{K}_{n+1}} W_{i}\right) /\left(\bigoplus_{i \in \mathcal{K}_{n+1} \backslash\left\{i_{n+1}\right\}} W_{i}\right) \cong$ $W_{i_{n+1}}$. Also, we get $V_{n+1} \oplus\left(\bigoplus_{i \in \mathcal{K}_{n+1} \backslash\left\{i_{n+1}\right\}} W_{i}\right) \oplus\left(\bigoplus_{i \in \mathcal{I}_{n} \backslash \mathcal{K}_{n+1}} W_{i}\right)=\left(\bigoplus_{i \in \mathcal{K}_{n+1}} W_{i}\right)$ $\oplus\left(\bigoplus_{i \in \mathcal{I}_{n} \backslash \mathcal{K}_{n+1}} W_{i}\right)$. This yields $V_{n+1} \oplus\left(\bigoplus_{i \in \mathcal{I}_{n+1}} W_{i}\right)=\bigoplus_{i \in \mathcal{I}_{n}} W_{i}$. Taking injective hulls of both sides, we get $E_{n+1} \oplus V_{n+1}=E_{n}$. Thus, we have $E=$ $E_{n+1} \oplus\left(\bigoplus_{k=1}^{n+1} V_{k}\right)$. Note that $\pi_{n}(L) \cap V_{n+1} \neq 0$ as it contains $C_{n+1}$. Thus, we have obtained a sequence of submodules $\left\{V_{j}\right\}, j=1,2, \ldots$, with the required properties. This completes the induction argument.

Now we claim that there exists a properly ascending chain $N_{0} \subset N_{1} \subset \ldots \subset$ $N_{j} \subset \ldots$ of submodules of $L$ such that $N_{0}=0$ and $E\left(N_{j} / N_{j-1}\right) \cong V_{j}$ for all $j \geq 1$.

Set $N_{j}=L \cap\left(V_{1} \oplus \ldots \oplus V_{j}\right)$. Clearly, $N_{0} \subseteq N_{1} \subseteq \ldots \subseteq N_{j} \subseteq \ldots$. Since $N_{j} \cap \operatorname{ker}\left(\pi_{j-1}\right)=N_{j-1}$, we have $N_{j} / N_{j-1} \cong \pi_{j-1}\left(N_{j}\right)$. If $l \in N_{j}$, then $l=$ $v_{1}+\ldots+v_{j}$ with $v_{i} \in V_{i}$, so $\pi_{j-1}(l)=v_{j}$ and $v_{j} \in \pi_{j-1}(L) \cap V_{j}$. This shows that $\pi_{j-1}\left(N_{j}\right) \subseteq \pi_{j-1}(L) \cap V_{j}$. Conversely, if $v_{j} \in \pi_{j-1}(L) \cap V_{j}$, then $v_{j}=\pi_{j-1}(l)$ with $l \in L \cap\left(V_{1} \oplus \ldots \oplus V_{j}\right)=N_{j}$, so $v_{j} \in \pi_{j-1}\left(N_{j}\right)$. Therefore $\pi_{j-1}\left(N_{j}\right)=\pi_{j-1}(L) \cap V_{j} \neq$ 0 . Because $\pi_{j-1}\left(N_{j-1}\right)=0$ and $\pi_{j-1}\left(N_{j}\right) \neq 0$, it follows that $N_{j-1} \subsetneq N_{j}$. Since $N_{j} / N_{j-1} \cong \pi_{j-1}\left(N_{j}\right)=\pi_{j-1}(L) \cap V_{j}$, we have $E\left(N_{j} / N_{j-1}\right) \cong V_{j}$.

Since $\left\{V_{j}: j \in \mathbb{N}\right\}$ is an independent family of uniform injective modules isomorphic to submodules of $M$, by the above lemma there exists an infinite subset $\mathcal{J} \subseteq \mathbb{N}$ such that $\bigoplus_{j \in \mathcal{J}} V_{j}$, and hence $\bigoplus_{j \in \mathcal{J}} E\left(N_{j} / N_{j-1}\right)$ is injective. Set $N=\bigcup_{j \in \mathcal{J}} N_{j}$. Given $j \in \mathcal{J}$, the canonical map $N_{j} \longrightarrow N_{j} / N_{j-1} \subset E\left(N_{j} / N_{j-1}\right)$ induces a map $\alpha_{j}: N \longrightarrow E\left(N_{j} / N_{j-1}\right)$. Let $\alpha: N \longrightarrow \bigoplus_{j \in \mathcal{J}} E\left(N_{j} / N_{j-1}\right)$ be defined by $\alpha(x)=\left\{\alpha_{j}(x)\right\}_{j \in \mathcal{J}}$ for all $x \in N$. Since $\bigoplus_{j \in \mathcal{J}} E\left(N_{j} / N_{j-1}\right)$ is injective, we may extend $\alpha$ to $\alpha^{*}: L \longrightarrow \bigoplus_{j \in \mathcal{J}} E\left(N_{j} / N_{j-1}\right)$. As $L$ is finitely generated, there exists a finite subset $\mathcal{K} \subseteq \mathcal{J}$ such that $\alpha^{*}(L) \subseteq \bigoplus_{k \in \mathcal{K}} E\left(N_{k} / N_{k-1}\right)$. For $j \in \mathcal{J} \backslash \mathcal{K}$ and $x \in N_{j}$ we have $0=\alpha_{j}(x)=x+N_{j-1}$, showing that $N_{j-1}=N_{j}$, a contradiction. 
Therefore, $M^{(\lambda)}$ is injective for any cardinal $\lambda$, and hence $M$ is $\Sigma$-injective.

(a) $\Longrightarrow$ (b) is obvious.

This completes the proof of Theorem 2 .

As a consequence of Theorem 2, we have the following characterization for a right noetherian ring.

Theorem 4. Let $R$ be a ring. Then the following are equivalent:

(i) $R$ is right noetherian.

(ii) For each injective module $M_{R}$, every essential extension of $M^{\left(\aleph_{0}\right)}$ is a direct sum of injective modules.

Proof. (i) $\Rightarrow$ (ii) is obvious. (ii) $\Rightarrow$ (i) follows from Theorem 2 and by Faith-Walker [5] that a ring $R$ is right noetherian if and only if every injective right $R$-module is $\Sigma$-injective.

Remark 5. The above result generalizes a result of Beidar-Ke 2] which states that a ring $R$ is right noetherian if and only if every essential extension of a direct sum of injective right $R$-modules is again a direct sum of injective right $R$-modules. Note that [2] indeed generalizes a result of Bass [1] that a ring is right noetherian if and only if every direct sum of injective modules is injective.

\section{ACKNOWLEDGMENT}

We would like to thank the referees for several helpful suggestions that simplified our proofs.

\section{REFERENCES}

[1] H. Bass, Finitistic dimension and a homological generalization of semiprimary rings, Trans. Amer. Math. Soc. 95 (1960), 466-488. MR0157984 (28:1212)

[2] K. I. Beidar and W.-F. Ke, On essential extensions of direct sums of injective modules, Archiv. Math. (Basel) 78 (2002), 120-123. MR1888412 (2003a:16006)

[3] A. Cailleau, Une caractérisation des modules $\Sigma$-injectifs, C. R. Acad. Sci. Paris Ser. A-B 269 (1969), A997-999. MR0260782(41:5405)

[4] C. Faith, Rings with ascending chain condition on annihilators, Nagoya Math. J. 27 (1966), 179-191. MR0193107 (33:1328)

[5] C. Faith and E. A. Walker, Direct-sum representations of injective modules, Journal of Algebra 5 (1967), 203-221. MR0207760(34:7575)

[6] J. M. Goursaud and J. Valette, Sur l'enveloppe des anneaux de groupes réguliers, Bull. Math. Soc. France 103 (1975), 91-102. MR0379569(52:474)

[7] C. Megibben, Countable injective modules are sigma-injective, Proc. Amer. Math. Soc. 84 (1982), 8-10. MR633266 (83a:16030)

Department of Mathematics, Ohio University, Athens, Ohio 45701

Department of Mathematics, Ohio University, Athens, Ohio 45701

E-mail address: jain@math.ohiou.edu

Department of Mathematics and Computer Science, St. Louis University, St. Louis, Missouri 63103

E-mail address: asrivas3@slu.edu 